OPEN ACCESS

Edited by:

Shokrollah Elahi

University of Alberta, Canada

Reviewed by:

Barbara Louise Lohman-Payne

University of Rhode Island,

United States

Zixin Wang

The Chinese University of Hong Kong,

China

${ }^{*}$ Correspondence: Veneranda M. Bwana

vbwana@nimr.or.tz

Specialty section: This article was submitted to

HIV and AIDS,

a section of the journal

Frontiers in Public Health

Received: 14 December 2017

Accepted: 23 April 2018

Published: 15 May 2018

Citation:

Bwana VM, Mfinanga SG,

Simulundu E, Mboera LEG and

Michelo C (2018) Accessibility of Early

Infant Diagnostic Services by Under-5

Years and HIV Exposed Children in

Muheza District, North-East Tanzania.

Front. Public Health 6:139

doi: 10.3389/fpubh.2018.00139

\section{Accessibility of Early Infant Diagnostic Services by Under-5 Years and HIV Exposed Children in Muheza District, North-East Tanzania}

\author{
Veneranda M. Bwana ${ }^{1,2 \star}$, Sayoki Godfrey Mfinanga ${ }^{3}$, Edgar Simulundu ${ }^{4}$, \\ Leonard E. G. Mboera ${ }^{5}$ and Charles Michelo ${ }^{1,6}$
}

${ }^{1}$ School of Public Health, University of Zambia, Lusaka, Zambia, ${ }^{2}$ Amani Research Centre, National Institute for Medical Research, Muheza, Tanzania, ${ }^{3}$ Muhimbili Research Centre, National Institute for Medical Research, Dar es Salaam, Tanzania, ${ }^{4}$ Department of Disease Control, School of Veterinary Medicine, University of Zambia, Lusaka, Zambia, ${ }^{5}$ National Institute for Medical Research, Headquarters, Dar es Salaam, Tanzania, ${ }^{6}$ Strategic Centre for Health Systems Metrics and Evaluations, School of Public Health, University of Zambia, Lusaka, Zambia

Introduction: Early infant diagnosis (EID) of Human Immunodeficiency Virus (HIV) provides an opportunity for follow up of HIV exposed children for early detection of infection and timely access to antiretroviral treatment. We assessed predictors for accessing HIV diagnostic services among under-five children exposed to HIV infection in Muheza district, Tanzania.

Methods: A cross sectional facility-based study among mother/guardian-child pairs of HIV exposed children was conducted from June 2015 to June 2016. Using a structured questionnaire, we collected information on HIV status, socio-demographic characteristics and other relevant data. Multiple regression analyses were used to investigate associations of potential predictors of accessing EID services.

Results: A total of 576 children with their respective mothers/guardians were recruited. Of the 576 mothers/guardians, 549 (95.3\%) were the biological mothers with a median age of 34 years (inter-quartile range: $30-38$ years). The median age of the 576 children was 15 months (inter- quartile range: 8.5-38.0 months). A total of 251 (43.6\%) children were born to mothers with unknown HIV status at conception. Only 329 (57.1\%) children accessed EID between 4 and 6 weeks of age. Children born to mothers with unknown HIV status at conception (AOR $=0.6,95 \% \mathrm{Cl} 0.4-0.8$ ) and those with ages 13-59 months (AOR $=0.4,95 \% \mathrm{Cl} 0.2-0.6$ ) were the significant predictors of missed opportunity to access EID. Children living with the head of household with at least a high education level had higher chances of accessing EID (AOR $=1.8,95 \% \mathrm{Cl} 1.1-3.3$ ). Their chances of accessing EID services was three-fold higher among mothers/guardians with good knowledge of HIV infection prevention of mother to child transmission $(\mathrm{AOR}=3.2,95 \% \mathrm{Cl} 2.0-5.2)$ than those with poor knowledge. Mothers/guardians living in rural areas had poorer knowledge of HIV infection prevention of mother to child transmission ( $\mathrm{AOR}=0.6,95 \% \mathrm{Cl} 0.4-0.9$ ) than those living in urban areas. 
Conclusion: Accessibility of EID services among children below 5 years exposed to HIV infection in Muheza is low. These findings stress the need for continued HIV education and outreach services, particularly in rural areas in order to improve maternal and child health.

Keywords: accessibility, children, early infant diagnosis, HIV, services, Tanzania

\section{INTRODUCTION}

Worldwide, human immunodeficiency virus (HIV) is considered as one of the most important infectious pathogen afflicting children. In 2016 it was estimated that 2.1 million children were living with HIV and more than $90 \%$ were in sub-Saharan Africa (SSA) (1). In the same year, approximately 160,000 new HIV infections, which occurred in children worldwide, were due to mother to child transmission of HIV (MTCT), either during pregnancy, childbirth or breast feeding (1). Most infected infants and children die from HIV-related causes without being tested of the infection. Without access to cotrimoxazole prophylaxis, antiretroviral therapy (ART) and supportive care, about $40 \%$ of HIV-infected infants in developing countries progress to death within 1 year of age and $50 \%$ within 2 years of age $(2,3)$. Studies have demonstrated a remarkable increase in survival rate if HIV-infected children have access to early HIV diagnosis and treatment (4).

Early Infant Diagnosis (EID) of HIV infection is part of maternal and child health service package that has been integrated into Prevention of Mother to Child Transmission of HIV (PMTCT) interventions since 2006 in most countries in SSA including Tanzania (5-9). EID using deoxyribonucleic acid-polymerase chain reaction (DNA-PCR) testing for HIV infection provides a definitive diagnosis in children less than 18 months of age $(10,11)$. The World Health Organization (WHO) recommends early testing for HIV DNA between 4 and 6 weeks of age to all infants born to HIV positive mothers $(8,12)$. EID provides an opportunity to identify HIV infected children for early clinical evaluation, prophylaxis for opportunistic infections and antiretroviral therapy (ART) aiming to reduce morbidity and mortality (4). However, late diagnosis of pediatric HIV infections and persistence of vertical transmission continues to be the major challenge despite the introduction of PMTCT services. In 2014, WHO estimated that only $50 \%$ of all HIV-exposed infants accessed EID services within 2 months of age (3). This is still far below the $80 \%$ EID services coverage recommended by WHO (8). These statistics suggest that accessibility to EID programs is still a challenge in resource-limited settings (13). Majority of HIVexposed and HIV-infected children remain unidentified due to inaccessibility and insufficient utilization of these services (14).

\footnotetext{
Abbreviations: AIDS, acquired immunodeficiency syndrome; ANC, antenatal care; ART, antiretroviral therapy; CTC, HIV Care and Treatment Centres; DNA, deoxyribonucleic acid; DBS, dried blood spots; EID, Early infant diagnosis; HIV, Human immunodeficiency virus; PSU, primary sampling units; KCMC, Kilimanjaro Christian Medical Centre; NACP, National AIDS Control Program; PCR, polymerase chain reaction; PMTCT, Prevention of mother to child transmission; RCH, Reproductive and child health; SSA, sub- Saharan Africa; TAT, turnaround time; WHO, World Health Organization.
}

According to a WHO report of 2016, MTCT accounts for about $18 \%$ of the new HIV infections in Tanzania (15). Good progress has been made in scaling up the quality of PMTCT services in Tanzania. More than 90\% of Reproductive and Child Health (RCH) clinics are providing PMTCT services (12). The Tanzania National AIDS Control Program (NACP) started HIV care and treatment services in 2004. It was estimated that more than 300,000 children below 15 years old were living with HIV in 2013 and only $16 \%$ of children estimated to be eligible for ART were receiving treatment by 2013 (16). The major contributing factors to the low ART coverage in infants and children in the country might be due to limited capacity of most health facilities to conduct virological testing (17). Nevertheless, the goal of EID is to identify early HIV infection prior to progression of clinical disease and referral of all HIV infected infants to HIV Care and Treatment Centres (CTC) (12). This is being done by collecting dried blood spots (DBS), which are then sent to four zonal laboratories located within referral hospitals, namely Kilimanjaro Christian Medical Centre (KCMC), Muhimbili National Hospital, Mbeya Referral Hospital and Bugando Medical Centre to be tested for HIV infection using DNA-PCR testing as part of EID services (18). By December 2016, there were 4,737 sites providing EID services in Tanzania scaled up to involve urban and rural areas in all regions (19).

Studies in Mozambique and Tanzania have shown that only 25 and $67 \%$ of infants who were registered for care were brought for EID, respectively $(20,21)$. In Mozambique about a quarter of HIV exposed infants received the first HIV test at a median age of 5.0 months (21) while in Zambia nearly three quarters received the test at a median age of 8.1 months (22). Accordingly, the median age at first HIV test was 5.0 months in Uganda (23) while in Cameroon it ranged from 1.5 to 4.0 months $(24,25)$. In contrast, in Nigeria, the median age was 13 weeks (range $=4.0-$ 72 weeks) (6) while studies in Tanzania reported the median age of 5.6-16.0 weeks at first HIV test $(9,20,26)$.

Overall, these studies suggest the existence of some variability at the age of first HIV test among children within the African region (27). In previous studies, stock out of supplies, limited laboratories that can perform PCR analysis, weak infrastructures and inadequate health personnel trained in DBS techniques were identified as challenges for effective EID services implementation in most countries in SSA (28-30). Maternal awareness of HIV control and prevention, cost of transport, poverty, stigma and discrimination $(5,7,28,31)$ were reported to affect access to EID services. Therefore, the main gaps concerning EID services are low coverage and timely testing at first HIV test as compared to $80 \%$ coverage and testing at the age of 4-6 weeks, respectively, as recommended by WHO. Consequently, EID services are 
still challenging in resource-limited countries in SSA including Tanzania, where only a few HIV exposed infants are tested $(3,21$, 32). This study aimed to assess predictors of mothers/guardians to obtain EID services for children aged under 5 years exposed to HIV infection in Muheza district, Tanzania. In the context of this study, a guardian was defined as the child's main primary caregiver living with the child in the same household and this included either the biological parent, grandparent, sister, brother, aunt or uncle.

\section{MATERIALS AND METHODS}

\section{Study Area}

The study was conducted in Muheza district north-eastern Tanzania $\left(4^{\circ}, 45^{\prime} \mathrm{S} ; 39^{\circ} 00^{\prime} \mathrm{E}\right)$. The district has a total of 46 health facilities (one district hospital, 4 health centers and 41 dispensaries); 42 of which provide RCH services, 36 offer PMTCT services and 28 offer EID services (33). The prevalence of HIV in the district as reported in 2013 was 3.9\% (34). The HIV prevalence among pregnant women in Muheza district in 2015 was $4.0 \%$ higher than that of its neighboring districts as detailed in the Supplementary Material (35). Muheza district was selected for this study because it is among the leading districts in Tanga Region with high HIV prevalence among pregnant women (35). The study was conducted in 18 health facilities (one district hospital, three health centers and 14 dispensaries).

\section{Study Design, Population and Sampling}

This was a cross-sectional study using a multi-stage sampling approach conducted during the period June 2015 to June 2016. In the first step, we aimed to select a total of 18 out of 46 health facilities in the district as detailed in the Supplementary Material. The health facilities defined the primary sampling units (PSUs)/clusters. A list of 46 PSUs was obtained from Muheza District Council and the facilities were numbered from one to 46 according to their geographical location. Then the sampling interval was obtained and 18 health facilities were randomly selected from the list. During the selection process, health facilities that were selected from the list and were found to have no EID services were removed and the next on the list was included. The process was repeated until all 18 facilities that were required for the study were included. The study population included all selected mothers/guardians with children below 5 years born to HIV positive mothers who were not breast feeding for $\geq 6$ weeks. The sample size was calculated based on the formula that accounted for simple random sampling and the design effect which accounted for between and within cluster variation (36). We assumed the highest exposure of infection risk and a response rate of $90 \%$ and thus the estimated sample size was 836 children.

\section{Data Collection}

Four nurse counselors were trained on how to conduct interviews using a pre-tested questionnaire. Most of the questions were closed with an open option, and some were open-ended. Information on HIV status, socio-demographic characteristics and other relevant family and health status were collected. Sociodemographic information included age, sex, religion, residence, income, education, marital status, occupation, and size of household. Further information collected included knowledge on HIV transmission and prevention, time period when the child's first HIV test ought to be performed, distance to the nearest health facility (which was defined by time taken in minutes to reach the facility on foot) and maternal information which included whether the index pregnancy was planned or not, as well as HIV status prior to conception.

Mothers/guardians' satisfaction on services at health facility was assessed based on five key questions. The questions were mainly addressing the mother/guardian's perceptions regarding health care services such as HIV testing, treatment, advice, nutritional and social support received at the health facility. Each question had five options of pre-coded responses with a neutral point being neither satisfied nor dissatisfied, or neither poor nor good. Moreover, the general quality of all health services was assessed on how low or high they were satisfied with the overall quality of health care services provided at a particular health facility. This included services as a whole, reception by health workers, comfortability with the environment of care, freedom to interact with health personnel (asking questions for clarification), family planning, adequate medicines and diagnostic services. Mother/guardian's willingness to receive the HIV testing and diagnosis for their children was also assessed.

Mother/guardian's knowledge and beliefs on HIV prevention and transmission including MTCT of HIV was also assessed based on four key questions. These questions were mainly addressing general HIV transmission, timing of post exposure prophylaxis to HIV exposed infant, factors affecting HIV transmission and prevention on the risk of MTCT. Mothers/guardians whose children received an HIV test late (i.e., testing at $\geq 7$ weeks of age) were asked to state the reasons for the delay. Multiple responses regarding the factors associated with missed opportunity to receive the first HIV test for their children at the age between 4 and 6 weeks were assessed. During recruitment, more data of the child and her mother were extracted from registers, child card and hospital case files to supplement the collected primary data.

\section{Laboratory Tests}

The HIV DNA PCR testing was used to confirm HIV infection in all HIV exposed children less than 18 months of age according to national guidelines. DBS samples were routinely collected from HIV exposed children at the first encounter to the health facility. For infants, using a heel prick, five circles were filled with blood on a specific Whatman filter paper card and air dried for $\geq 4 \mathrm{~h}$ on a drying rack. After drying, the cards were placed in a gasimpermeable zip locked bag with desiccant sachets and stored in a safe location. All DBS samples from peripheral health centers and dispensaries were transported to the district hospital and then were mailed to the KCMC Clinical Laboratory in Moshi, Tanzania. In the laboratory, the DBS were tested for HIV DNA using version two of the COBAS AmpliPrep/COBAS TaqMan 48 system. One DBS circle was used to run a DNA-PCR test and if positive, a second circle was analyzed to confirm the 
first result. A test result was considered positive if both PCR assays were positive. The Rapid HIV antibody test was done in all HIV exposed children aged more than 18 months using Determine ${ }^{\circledR} /$ Bioline ${ }^{\circledR}$ rapid tests for the first test. If the first test was positive, the second test was performed using Uni-Gold ${ }^{\circledR}$ rapid test. HIV diagnosis was confirmed based on concordance of the results of these two HIV rapid tests. Furthermore, the sample turnaround time (TAT) which was defined as time between DBS sample collection and return of HIV DNA PCR results to the facility, for the first HIV DNA PCR test was assessed.

\section{Data Management and Analysis}

To facilitate data entry, responses provided in open-ended questions were re-coded into themes which were developed to respond to study objectives. Data were double entered in Epi Data database version 3.1 (http://www.epidata.dk/) and then transferred to STATA version 13 statistical package (Stata Corp, College Station, Texas, USA). Continuous variables were described using median and inter quartile range (IQR). Categorical variables were described using frequencies and percentages. The accessibility to first HIV test by the child was categorized into a binary variable (dependent variable), "yes" as obtained or "no" as not obtained the test at the age of 4-6 weeks. A composite variable on knowledge and beliefs on HIV prevention and MTCT of the mother/guardian was generated based on the four key questions as described above. The knowledge level was categorized into a binary variable "good" or "poor." Guardians' perceptions to healthcare services based on five options of pre-coded responses with a neutral point being neither satisfied nor dissatisfied, or neither poor nor good were described by using percentages.

Binary logistic regression was done and all factors with $p$ values of $\leq 0.2$ including priori factors were considered for multiple variable logistic regression analysis. Multiple logistic regression analyses were used to examine the associations between various factors (including child and maternal, guardian, head of household characteristics) and service accessibility by the child at the age of 4-6 weeks. A manual backward stepwise selection was employed by removing non-significant variables (one at a time starting with those with the highest $p$-value). Goodness of fit of the final model was tested using likelihood ratio test. The final model consisted of variables that were significant at $p$-value of $\leq 0.05$ including those with epidemiological importance. Adjusted odds ratios with their corresponding 95\% confidence intervals were estimated and presented. Three individual multiple regression models were fitted for (1) the child and maternal variables, (2) guardian variables, and (3) the head of household variables. Subsequently, two combined models were fitted: (1) a model that contains the three set variables; and (2), a model that includes only significant variables which was used in the final interpretation. In addition, a separate model to assess the mother/guardian's knowledge of HIV (including MTCT and PMTCT) was also fitted. In this study, "accessibility of first HIV test by the child at the age of 4-6 weeks" was defined as the time when the child was obtaining HIV test at the age of 4-6 weeks.

\section{Ethical Considerations}

Ethical approval was obtained from the Medical Research Coordinating Committee of the National Institute for Medical Research in Tanzania (Ref: NIMR/HQ/R.8a/Vol. IX/1978) and University of Zambia Biomedical Research Ethics Committee (Ref: 001-01-15). Permission to conduct this study was given by Muheza District Council Authority. Written consent was obtained from each guardian before recruitment. Counseling of the mothers/guardians of HIV-exposed children before and after the HIV test was performed in accordance with standard testing and counseling guidelines. In this study the HIV status for all HIV exposed children was determined for effective appropriate care and management. All confirmed HIV positive children were referred to CTC for initiation of ART in accordance with Tanzania guidelines on the management of HIV and AIDS.

\section{RESULTS}

\section{Baseline Characteristics}

A total of 576 mother/guardian-child pairs were included in the study. The median age of the mothers/guardians included in the study was 34 years (IQR: 30-38 years). Majority (95.3\%) were the biological mothers. Thirteen $(2.3 \%)$ children were living with grandmothers as their guardians. Most $(70.1 \%)$ of the head of household were reported to be males. Of the children included, $51.2 \%$ were females. The median age was 15 months (IQR: 8.5-38). A total of 329 (57.1\%) children received the first HIV test between 4 and 6 weeks of age. The overall median age at the time of first HIV test was 6 weeks (IQR: 6-20 weeks). Sixty-one $(10.6 \%)$ children were found to be HIV- positive of which 31 were confirmed by HIV DNA test, and 30 by HIV antibody test. Majority $(67.2 \%, n=41)$ of HIV positive children were diagnosed at $\geq 13$ months of age. Of the $61 \mathrm{HIV}$ positive children, the overall median age at diagnosis was 20 months (IQR: 12.5-35 months). Accordingly, four (6.5\%), 15 (24.6\%), and $42(68.9 \%)$ HIV positive children were $\leq 12$ months, $13-$ 24 months and 25-59 months age group, respectively. Out of 576 children, $43.6 \%, n=251$ ) were born to mothers with unknown HIV status at conception. A proportion of women $(5.9 \%, n=34)$ in this study never had an HIV test before and even during their index pregnancy. Some $(1.9 \%, n=11)$ mothers were tested after their sick children tested positive when seeking care at a health facility. Nevertheless, a number of these mothers did not know their HIV status before being pregnant and were labeled as "unknown HIV status at conception" (Table 1).

\section{Turnaround Time}

Out of 576 children, $88.5 \%(n=510)$ children received the first HIV DNA PCR test. Only 413 records had complete data on the date of sample collection and the date of arrival of the DNA PCR results to the health facility. The date of arrival of PCR results to health facility was not recorded in 97 records. The sample TAT for first HIV DNA test for the 413 records examined was 6 weeks (IQR: 5-10 weeks). In addition, some mothers/guardians were not satisfied with the receipts 
TABLE 1 | Demographic distribution of the study participants.

\begin{tabular}{|c|c|}
\hline Children & $n(\%)$ \\
\hline \multicolumn{2}{|l|}{ Age category(months) } \\
\hline$\leq 12$ & $224(38.9)$ \\
\hline $13-24$ & $155(26.9)$ \\
\hline $25-59$ & $197(98.8)$ \\
\hline \multicolumn{2}{|l|}{ Sex } \\
\hline Male & $281(48.8)$ \\
\hline Female & $295(51.2)$ \\
\hline \multicolumn{2}{|l|}{ Residence } \\
\hline Rural & $445(77.3)$ \\
\hline Urban & $131(22.7)$ \\
\hline \multicolumn{2}{|l|}{ Guardians } \\
\hline \multicolumn{2}{|l|}{ Age category(years) } \\
\hline $15-24$ & $58(10.1)$ \\
\hline $25-70$ & $518(89.9)$ \\
\hline \multicolumn{2}{|l|}{ Sex } \\
\hline Male & $7(1.2)$ \\
\hline Female & $569(98.8)$ \\
\hline \multicolumn{2}{|l|}{ Education } \\
\hline No education & 78 (13.5) \\
\hline Primary complete & $451(78.3)$ \\
\hline Secondary/high school and above & $47(8.2)$ \\
\hline \multicolumn{2}{|l|}{ Marital status } \\
\hline Married/living together & $519(90.1)$ \\
\hline Separated/divorced/widow & 57 (9.9) \\
\hline \multicolumn{2}{|l|}{ Occupation } \\
\hline Trading & $97(16.8)$ \\
\hline Formal employment & $15(2.6)$ \\
\hline Subsistence farmer & $464(80.6)$ \\
\hline \multicolumn{2}{|l|}{ Income } \\
\hline Low ( $\leq 34$ US Dollar) & $45(7.8)$ \\
\hline High (>34 US Dollar) & $531(92.2)$ \\
\hline \multicolumn{2}{|l|}{ Relation to the child } \\
\hline Mother & 549 (95.3) \\
\hline Father & $7(1.2)$ \\
\hline Grandmother & $13(2.3)$ \\
\hline Aunt & $7(1.2)$ \\
\hline \multicolumn{2}{|l|}{ Heads Of Household } \\
\hline \multicolumn{2}{|l|}{ Age category (years) } \\
\hline $15-24$ & $31(5.4)$ \\
\hline $25-85$ & $545(94.6)$ \\
\hline \multicolumn{2}{|l|}{ Sex } \\
\hline Male & $404(70.1)$ \\
\hline Female & $172(29.9)$ \\
\hline \multicolumn{2}{|l|}{ Education } \\
\hline No education & $58(10.0)$ \\
\hline Primary complete & $46(8.0)$ \\
\hline Secondary/high school and above & $474(82.0)$ \\
\hline \multicolumn{2}{|l|}{ Relation to the child } \\
\hline Mother & 65 (11.3) \\
\hline Father & $355(61.6)$ \\
\hline Grandmother/grandfather & $131(22.7)$ \\
\hline Other relatives ${ }^{\ddagger}$ & $25(4.4)$ \\
\hline
\end{tabular}

(a) Total sample size, $N=576$. (b) Guardian in this study was the main primary caregiver of the child living in the same household. (c) ${ }^{\ddagger}$ Other relatives included were the sister, brother, aunt and uncle. of HIV results for their children due to variability in TAT. Some $(12.5 \%, n=72)$ were discouraged to return to a health facility to collect their children's results. Three $(0.5 \%)$ guardians each with HIV exposed child aged 6 months, 9 months, and 4 years, respectively declared that they have never received the first DBS results of their children since birth. Others $(1.0 \%$, $n=6$ ) claimed that their results were not seen or found at the clinic.

\section{Predictors Associated With Accessibility of EID Services}

In multiple logistic regression models for individual characteristics, we found that children with mothers/guardians who were married/living together with their spouses, having general good knowledge on HIV prevention and transmission from mother to child were statistically significant predictors of accessing EID at the age of 4-6 weeks. Additionally, children with the head of household as the father who had attained secondary or high school education were significantly associated with timely access to EID services (Table 2). Mothers/guardians with good knowledge on HIV, had significantly higher odds ratio for the mother/guardian-child pairs accessing $\mathrm{EID}(\mathrm{AOR}=3.0$, 95\% CI 1.7-4.8) compared to those with poor knowledge. For every mother/guardian who was married/living together with their spouses, the odds ratio of mother/guardian-child pairs accessing EID increased (AOR $=2.3$, 95\% CI 1.2-4.6) compared to those who were separated/divorced. Those with the head of household as the father (AOR $=2.1,95 \% \mathrm{CI}$ 1.2-3.6) and who has attained secondary or high school education $(\mathrm{AOR}=1.8,95 \%$ CI 1.2-3.3), had significantly higher odds for mother/guardian-child pairs accessing EID (Table 2).

In contrast, having a child in the age groups of 13-24 months $(\mathrm{AOR}=0.4,95 \%$ CI $0.2-0.6)$ or 25-59 months $(\mathrm{AOR}=0.3,95 \% \mathrm{CI} 0.2-0.5)$, and a child being found HIV positive $(\mathrm{AOR}=0.2,95 \% \mathrm{CI} 0.1-0.4)$ were associated with lower odds of timely access to EID services (i.e., they did not obtain HIV test at 4-6 weeks of age). Similarly lower odds to access EID were observed among children born to mothers with unplanned pregnancies $(\mathrm{AOR}=0.7,95 \% \mathrm{CI} 0.5-0.9)$ and those born to mothers with unknown HIV status at conception $(\mathrm{AOR}=0.6$, 95\% CI 0.4-0.8). Likewise, lower odds to access EID were also observed among children with mothers/guardians who did not know the age when the first HIV test of the child ought to be performed $(\mathrm{AOR}=0.2,95 \% \mathrm{CI} 0.1-0.3)$; and those who lived in areas located far away from a health facility $(\mathrm{AOR}=0.5,95 \% \mathrm{CI}$ $0.3-0.7$ ) (Table 2).

However, in a separate multiple variable analysis that combined all variables, we found that children with mothers/guardians who were married/living together with their spouses $(\mathrm{AOR}=2.3,95 \% \mathrm{CI} 1.2-4.6)$, having general good knowledge on HIV (AOR $=2.4$, 95\% CI 1.4-4.0) remained independently associated with higher chances of accessing EID services. Having a child in the age groups of 13-59 months (AOR $=0.4,95 \%$ CI $0.2-0.7$ ), a child being found HIV positive $(\mathrm{AOR}=0.3,95 \% \mathrm{CI} 0.1-0.6)$, living far away from the health 
TABLE 2 | Multiple logistic regression of predictors of HIV testing of children at the age of 4-6 weeks in Muheza by.

\begin{tabular}{|c|c|}
\hline Variables & AOR(95\% Cl) \\
\hline \multicolumn{2}{|l|}{ (i) Child and maternal characteristics } \\
\hline \multicolumn{2}{|l|}{ Age category(months) } \\
\hline$\leq 12$ & 1 \\
\hline $13-24$ & $0.4(0.2-0.6)^{\star}$ \\
\hline $25-59$ & $0.3(0.2-0.5)^{\star}$ \\
\hline \multicolumn{2}{|l|}{ Sex } \\
\hline Female & 1 \\
\hline Male & $1.0(0.7-1.5)^{\star \star}$ \\
\hline \multicolumn{2}{|l|}{ Residence } \\
\hline Urban & 1 \\
\hline Rural & $1.1(0.7-1.8)^{\star \star}$ \\
\hline \multicolumn{2}{|l|}{ Place of delivery } \\
\hline Home & 1 \\
\hline Health facility & $0.6(0.3-1.2)^{\star \star}$ \\
\hline \multicolumn{2}{|l|}{ HIV status } \\
\hline Negative & 1 \\
\hline Positive & $0.2(0.1-0.4)^{*}$ \\
\hline \multicolumn{2}{|l|}{ Maternal HIV status at conception } \\
\hline Known & 1 \\
\hline Unknown & $0.6(0.4-0.8)^{\star}$ \\
\hline \multicolumn{2}{|l|}{ Maternal planned index pregnancy } \\
\hline Yes & 1 \\
\hline No & $0.7(0.5-0.9)^{*}$ \\
\hline Unknown & $0.4(0.1-1.3)^{\star \star}$ \\
\hline \multicolumn{2}{|c|}{$\begin{array}{l}\text { (a) *Significant variables based on P-value, }{ }^{* *} \text { Not significant at } P \text {-value } \leq 0.05 \text {. (b) } \\
\text { AOR, Adjusted odd ratio; Cl, Confidence interval. }\end{array}$} \\
\hline Variables & AOR(95\% Cl) \\
\hline \multicolumn{2}{|l|}{ (ii) Guardian characteristics } \\
\hline \multicolumn{2}{|l|}{ Age category(years) } \\
\hline $25-70$ & 1 \\
\hline $15-24$ & $1.1(0.6-2.3)^{\star \star}$ \\
\hline \multicolumn{2}{|l|}{ Education } \\
\hline No education & 1 \\
\hline Primary complete & $0.7(0.4-1.3)^{\star \star}$ \\
\hline Secondary/high school and above & $0.9(0.4-2.5)^{\star \star}$ \\
\hline \multicolumn{2}{|l|}{ Marital status } \\
\hline Separated/divorced/widow & 1 \\
\hline Married/living together & $2.3(1.2-4.6)^{\star}$ \\
\hline \multicolumn{2}{|l|}{ Reported age to perform first test } \\
\hline 4-6 weeks & 1 \\
\hline$\leq 1$ week, 2-240 weeks & $0.07(0.04-0.13)^{\star \S}$ \\
\hline I don’t know & $0.2(0.1-0.3)^{\star}$ \\
\hline \multicolumn{2}{|l|}{ Knowledge on HIV } \\
\hline Poor & 1 \\
\hline Good & $3.0(1.7-4.8)^{\star}$ \\
\hline \multicolumn{2}{|l|}{ Monthly income } \\
\hline High (>34 US Dollar) & 1 \\
\hline Low ( $\leq 34$ US Dollar) & $1.6(0.7-3.7)^{\star \star}$ \\
\hline \multicolumn{2}{|l|}{ Size of the household } \\
\hline$\leq 7$ people & 1 \\
\hline$>7$ people & $0.5(0.2-0.9)^{\star}$ \\
\hline \multicolumn{2}{|l|}{ Distance to health facility } \\
\hline Near ( $\leq 30 \mathrm{~min})$ & 1 \\
\hline $\operatorname{Far}(>30 \mathrm{~min})$ & $0.5(0.3-0.7)^{\star}$ \\
\hline
\end{tabular}

(a) *Significant variables at $P$-value $\leq 0.05$, ${ }^{* *}$ Not significant at $P$-value $\leq 0.05$. (b) $A O R$, Adjusted odd ratio; Cl, Confidence interval. (c) § Unable to round off.
TABLE 2 | Continued

\section{Variables}

$\operatorname{AOR}(95 \% \mathrm{Cl})$

\section{(iii) Head of household characteristics}

Age category (years)

25-85

15-24

Education

No education

Primary complete

Secondary/high school and above

Relation to the child

Mother

Father

Grandmother/grandfather

Other relatives ${ }^{\ddagger}$

(a) *Significant variables at $P$-value $\leq 0.05,{ }^{* *}$ Not significant at $P$-value $\leq 0.05$. (b) $A O R$, Adjusted odd ratio; Cl, Confidence interval. (c) ${ }^{\ddagger}$ Other relatives included were the sister, brother, aunt and uncle.

facility $(\mathrm{AOR}=0.6,95 \% \mathrm{CI} 0.4-0.9)$ and mothers/guardians who did not know the age when the first HIV test of the child ought to be performed ( $\mathrm{AOR}=0.2,95 \% \mathrm{CI} 0.1-0.4$ ) remained independently associated with lower chances to access EID services (Table 3).

\section{Knowledge of HIV Transmission and Prevention}

Results of the multiple logistic regression model (Table 4) showed significant association between general knowledge on HIV (including MTCT and PMTCT) and level of education, residence and marital status. The mothers/guardians who were married or living together with their spouses ( $\mathrm{AOR}=2.8,95 \%$ CI 1.6-4.9) and with a primary education ( $\mathrm{AOR}=1.8,95 \%$ CI 1.1-3.1) had good knowledge on HIV (including MTCT and PMTCT). Mothers/guardians living in rural areas had poor knowledge on HIV (including MTCT and PMTCT) $(\mathrm{AOR}=0.6$, 95\% CI 0.4-0.9).

\section{Associated Barriers to Access EID Services}

A total of 247 mothers/guardians whose children missed the first HIV test between 4 and 6 weeks of age were also interviewed. Factors that hindered access to EID services included inadequate knowledge and awareness of EID services (25.5\%), children appeared to be in good health (13.8\%) and so the guardians felt there was no need to access the services, lack of paternal permission or support (12.6\%) and some mothers feared the possibility of their children being found HIV positive (5.4\%). Stock out of DBS kits (6.5\%), unavailability of trained staff (2.0\%), poor DBS sample collection techniques $(2.8 \%)$ and lack of knowledge among health workers on the correct age when the first HIV test of the child is supposed to be performed (4.5\%) were found to affect access to EID services. In addition, three mothers refused to take ARV and said that they believed in the Almighty God who will cure them from HIV infection through prayers. Seventeen mothers did not inform their spouses about their HIV status. These attitudes and beliefs deterred women from 
TABLE 3 | Multiple logistic regression results of predictors of HIV testing of children at the age of 4-6 weeks in Muheza district, Tanzania.

\begin{tabular}{lc}
\hline Children & AOR(95\% Cl) \\
\hline Age category(months) & 1 \\
$\leq 12$ & $0.4(0.2-0.7)^{\star}$ \\
$13-24$ & $0.4(0.2-0.7)^{\star}$ \\
$25-59$ & \\
HIV status & 1 \\
Negative & $0.3(0.1-0.6)^{\star}$ \\
Positive & \\
Guardians & \\
Marital status & 1 \\
Separated/divorced/widow & $2.3(1.2-4.6)^{\star}$ \\
Married/living together & \\
Reported age to perform first test & 1 \\
$4-6$ weeks & \\
$\leq 1$ week, 2-240 weeks & $0.6(0.4-0.9)^{\star}$ \\
I don't know & $0.08(0.04-0.14)^{\star \S}$ \\
Knowledge on HIV & $0.2(0.1-0.4)^{\star}$ \\
Poor & \\
Good & $1.4-4.0)^{\star}$ \\
Fistance to health facility & \\
\hline & \\
\hline
\end{tabular}

(a) *Significant variables at $P$-value $\leq 0.05$. (b) $A O R$, Adjusted odd ratio; Cl, Confidence interval. (c) § Unable to round off.

seeking PMTCT services which led to poor adherence to PMTCT interventions including accessing EID services (Table 5).

\section{Satisfaction of Mothers/Guardians on Health Care Services}

A total of 576 guardians were asked about their satisfaction with the quality of services offered at health facilities. About 402 (69.8\%) mothers/guardians were satisfied with the level of care at the facility in terms of space, confidentiality and attention. Most of them $(85.5 \%, n=492)$ were satisfied with EID services provided to their children (Figure 1). Generally, low satisfaction with the overall quality of services at the facility was reported by 306 (53.1\%) mothers/guardians. However, 559 (97.1\%) mothers/guardians were willing to receive EID services for their children.

\section{DISCUSSION}

We observed that only slightly over half of the children below 5 years exposed to HIV infection accessed EID services between 4 and 6 weeks of age in Muheza district. The EID services coverage in Muheza is still below the $80 \%$ threshold recommended by WHO (8). However, the coverage has increased compared to previous reported data from Tanzania and some countries in SSA which ranged from 4 to $55 \%(24,27,37-39)$. Nevertheless, EID services coverage is considerably lower when compared to studies conducted in Botswana, Swaziland, South Africa and Malawi which ranged from 58 to $94 \%(27,38,40,41)$.
TABLE 4 | Guardian characteristics associated with general good knowledge on HIV transmission and prevention from mother to child.

\begin{tabular}{lc}
\hline Guardians & AOR(95\% Cl) \\
\hline Age category(years) & 1 \\
$25-70$ & $2.0(0.9-4.4)^{\star \star}$ \\
$15-24$ & \\
Sex & 1 \\
Female & $0.2(0.1-1.2)^{\star \star}$ \\
Male & \\
Residence & 1 \\
Urban & $0.6(0.4-0.9)^{\star}$ \\
Rural & 1 \\
Education & $1.8(1.1-3.1)^{\star}$ \\
No education & $0.7(0.3-1.5)^{\star *}$ \\
Primary complete & \\
Secondary or high school and above & 1 \\
Marital status & $2.8(1.6-4.9)^{\star}$ \\
Separated/divorced/widow & \\
Married/living together & \\
\hline
\end{tabular}

(a) ${ }^{*}$ Significant variables at $P$-value $\leq 0.05$, ${ }^{*}$ Not significant at $P$-value $\leq 0.05$. (b) $A O R$, Adjusted odd ratio; $\mathrm{Cl}$, Confidence interval.

Several factors affecting access to EID including both individual and health facility (institutional) were identified. At the individual level, inadequate maternal knowledge and awareness of EID services, lower levels of education of the head of household, lack of paternal support/permission, larger size of household, cost of transport, long distance to health facilities and HIV-related stigma were identified as barriers of accessing EID services. At the health facility level, unavailability of trained staff, inadequate supplies of laboratory materials and late return of HIV test results were the main constraining factors.

Older children and those who were HIV infected had accessed the first HIV test at $\geq$ to 7 weeks of age. The observed late accessibility to HIV testing and late HIV diagnosis in older children was also consistent with other studies $(42,43)$. This implies that accessibility to HIV testing among children at older ages could have been prompted by manifestation of clinical symptoms that required diagnostic testing. This suggests that the current recommended age to access EID should be evaluated and if feasible to start performing the first HIV test within few days after delivery. The strategy will create awareness and increase motivation to bring the child later at the age of 6 weeks and EID coverage will be increased. This will ensure early enrolment into continuum of care in order to identify early HIV infection for timely access to ARV treatment.

Children born to mothers with unknown HIV status at conception were among those who missed an opportunity to access EID services in Muheza. Several studies reported that routine HIV testing to all infants presented at vaccination clinics $(27,44-48)$ and to all hospitalized children $(49,50)$ have been found to identify large number of HIV exposed infants and children. In Botswana, Uganda and Malawi, EID of $\mathrm{HIV}$ is 
TABLE 5 | Barriers associated with accessibility to EID services in Muheza.

\section{Individual factors}

$n(\%)$

Culture issues $\#$

$11(4.5)$

Children appeared in good health

$34(13.8)$

Inadequate knowledge and awareness on EID services

$63(25.5)$

Long distance to the health facility

9 (3.6)

Lack of money for transport to go to the health facility

Lack of paternal permission/support to access EID services

$13(5.3)$

$31(12.6)$

$13(5.3)$

Guardian was uninformed if the child was born to HIV+

mother

Guardian-child pairs relocated to a residence far away from the facility with EID service

Their mothers disbelieve their HIV + results and were not on ARV during pregnancy ${ }^{\dagger}$

Mothers feared that their HIV+ status will be known by health workers*

Mothers feared if their children would be found to be HIV+ ${ }^{*}$

\section{Health facility factors}

Long waiting time to get services at the facility

Health workers did not know the correct age to perform

child's first HIV test

Mothers not registered at the health facility, so the child was

not tested

The child's clinic card did not have facility number, so he was

not tested

Unavailability of trained health workers

Stock out of DBS kits

Improper collection of DBS specimen

The first HIV test results lost

$10(4.0)$

(a) \#The cultural does not allow to send back the young child to the health facility, the cultural does not allow the young child to be carried by everyone and should be kept inside the house. (b) †Three mothers believe in Almighty God who will cure them from HIV infection through prayers and refuse to take ARV. (c) *HIV related stigma experienced by 17 mothers has directly affect access to EID services.

offered even to those infants born to mothers of unknown HIV status (51-53). In contrast, in Mozambique, EID programs target infants born to mothers with known HIV positive status (21). These studies indicate that a considerable number of HIV infected children will remain unidentified in the community if EID services are offered only to children born to mothers with known HIV status.

In our study, higher chances to access EID services were observed among children whose heads of household had attained high education level. Studies conducted elsewhere reported that parents with low educational status tend to have poor follow up to scheduled clinic visits $(28,54,55)$. In addition, inadequate mother/guardian's knowledge and awareness of HIV/AIDS observed in our study, have also been shown to affect access to EID in South Africa (56). Interestingly, a number of HIV exposed children were living with grandmothers as their main primary caregivers in Muheza. Similar finding have been reported elsewhere $(56,57)$. These grandmothers may be unaware of EID services or may not know the importance of regular child follow up to EID services. As such community awareness and understanding of the ongoing HIV and AIDS interventions programs should be emphasized to every individual, including grandmothers.

In our study, the long distance to the health facility, larger size of the household, affordability to cater for transport costs and paternal permission/support were found to affect access to EID. Similar factors have been reported elsewhere $(21,28,37,41,49,50,57,58)$. These findings suggest the need to develop and strengthen the health related funding mechanisms that will improve social networks and support structures in the community. In addition, HIV-related stigma, such as failure by a maternal spouse to disclose their HIV status to their family, maternal fear of the possibility of their child being found HIV positive were found to affect access to EID services in Muheza District. This results into poor compliance to PMTCT and EID services and may lead to poor attendance of regular clinic visits for their children. These findings were also similar to those reported previously (31, 55).

Health facility factors were also found to affect EID services in this study. Poor DBS sample collection techniques and lack of knowledge of the age when the first HIV test of the child ought to be performed among health workers were observed to affect EID. Stock out of laboratory materials like DBS kits was also a barrier to access EID services in the district. Similar findings have been documented in other studies $(28,30,39,55)$. Provision of continuous training especially in PMTCT and EID services to health personnel with first priority being given to those involved in providing pediatric care is highly needed. Furthermore, the long TAT of PCR results have been shown to affect access to EID services in our study. The finding is consistent with those reported from other studies $(22,23,25$, $28,47)$. The long TAT definitely leads to delay in making early diagnosis for early initiation of ART for HIV infected children, whilst continuing with routine care for HIV uninfected children.

This study has limitations, including the potential for reporting bias of the date of first HIV test and what transpired during the pregnancy of their index children. Of note, reporting bias was observed in those children who had received the first HIV test before initiation of our study. But this was minimized through verification using the children's under five or antenatal cards, the antenatal care (ANC) records, PMTCT records and laboratory DBS forms. The records were compared to the responses given by the respondents. Stratified random selection could have been the best approach in this study, but this would have entailed to enroll more facilities per strata, and considering the limited resources we opted not to stratify. A total of 28 guardians with HIV exposed children below 5 years refused to participate in the study. These mothers/guardians-child pairs may have different characteristics compared to those who participated in the study. Finally, while factors included in our multiple variables logistic model were each potentially important predictors of accessibility to EID services, we cannot eliminate the probability that our results were manipulated by unmeasured confounders. 


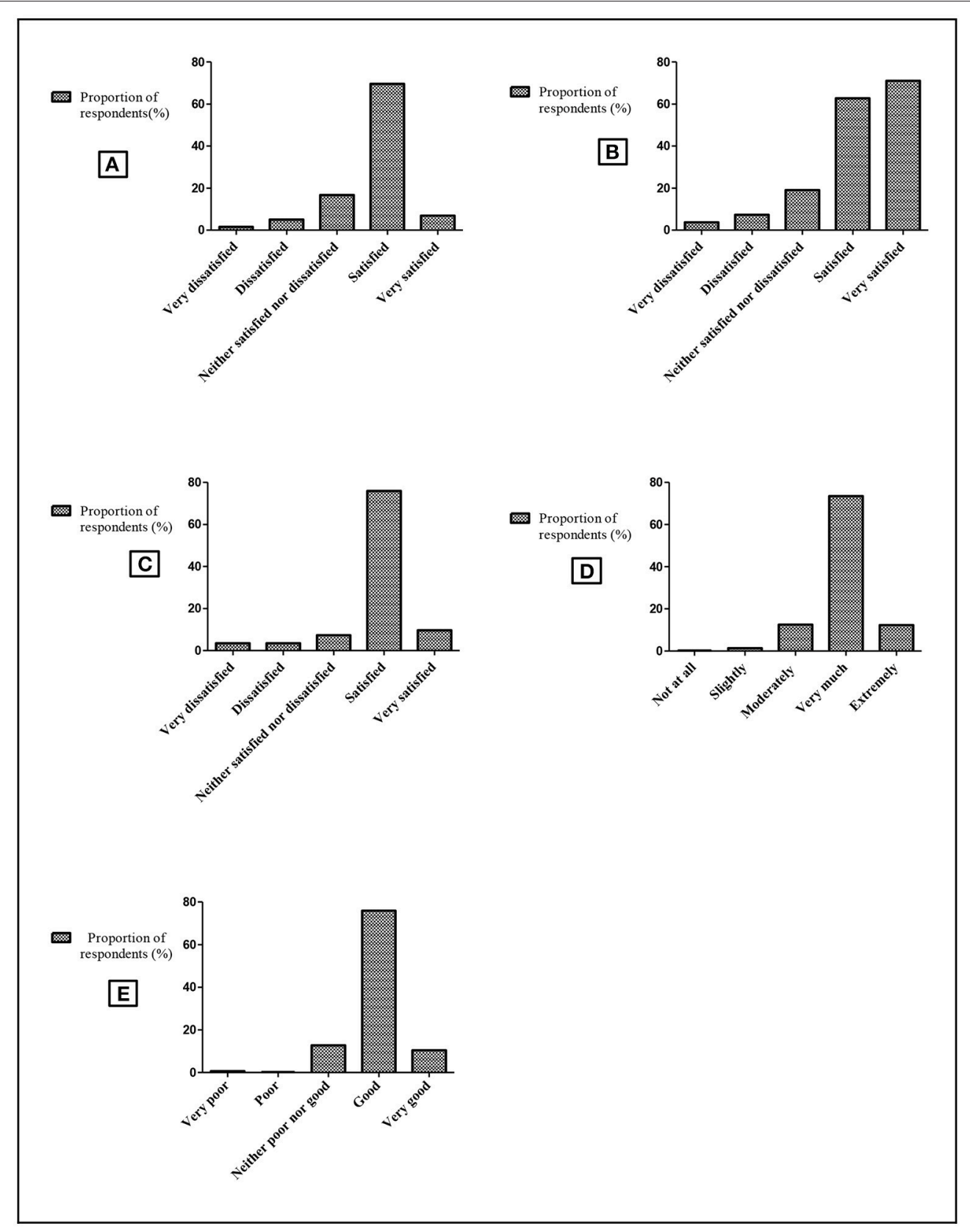

FIGURE 1 | Mothers/guardians' satisfaction by health care services in Muheza. Schematic presentation showing mother/guardian's perceptions regarding receiving services at the health facility such as level of care, HIV testing, treatment and advice. The horizontal axis shows satisfaction levels as a rating scale of five options. The vertical axis shows proportion of respondents (mothers/guardians) corresponding to the 5 options on each column. (A) Level of satisfaction with the reception given by health care workers. (B) Level of satisfaction of care at the facility (space, confidentiality, attention). (C) Level of satisfaction with EID services provided to their children. (D) Trustworthiness on health care workers' information (care, nutritional, social support). (E) Level of overall quality of health care services at the facility.

\section{CONCLUSION}

Accessibility to HIV testing and diagnostic services among children exposed to HIV infection in Muheza district is low despite the investment made in the past decade. This could be partly due to poor knowledge of PMTCT among mothers/guardians. The findings underscore the need to focus on effective implementation of PMTCT programs in order to improve EID services in similar settings. More strategies are needed to evaluate the age for entry point to access EID services in order to increase EID coverage. In addition, community awareness and education of the ongoing HIV and AIDS interventions programs, training of health workers especially in PMTCT and EID services should be improved. Educational 
campaigns that provide massages on the risk factors of MTCT and the importance of follow up appointments to EID clinics need to be emphasized.

\section{AUTHOR CONTRIBUTIONS}

VB conceived and conducted the study. VB, CM, LM, SM, ES contributed to the study design and analysis of results. CM, LM, SM, and ES were involved in supervision. VB drafted the manuscript. All authors contributed to revision and approval of the final version of the manuscript.

\section{FUNDING}

VB was sponsored by the Intra - ACP (African, Caribbean and Pacific) Academic Mobility Scheme (agreement no. 20123166/001-001) for her PhD studies at the University of Zambia.

\section{ACKNOWLEDGMENTS}

We would like to acknowledge the excellent technical and field assistance of Bernadina Ruta, Stella Kolugendo, Josephine Mhina, Irene Lazaro, Grades Stanley, Fagason Mduma, Veronica

\section{REFERENCES}

1. Joint United Nations Programme on HIV/AIDS. UNAIDS Data (2017). Available online at: http://www.who.int/hiv/pub/toolkits/ PMTCT9789241596015_eng.pdf

2. Newell M-L, Coovadia H, Cortina-Borja M, Rollins N, Gaillard P, Dabis F, et al. Mortality of infected and uninfected infants born to HIVinfected mothers in Africa: a pooled analysis. Lancet (2004) 364:1236-43. doi: 10.1016/S0140-6736(04)17140-7

3. World Health Organization. Towards Universal Access: Scaling Up Priority HIV/AIDS Interventions in the Health Sector. Progress Report 2010. World Health Organization (2010), p. 1-150. Availble online at: http://www.who.int/hiv/pub/2010progressreport/en/

4. Violari A, Cotton MF, Gibb DM, Babiker AG, Steyn J, Madhi SA, et al. Early antiretroviral therapy and mortality among HIV-infected infants. $N$ Engl J Med. (2008) 359:2233-44. doi: 10.1056/NEJMoa0800971

5. Torpey K, Mandala J, Kasonde P, Bryan-Mofya G, Bweupe M, Makundu J, et al. Analysis of HIV early infant diagnosis data to estimate rates of perinatal HIV transmission in Zambia. PLoS ONE (2012) 7:e42859. doi: 10.1371/journal.pone.0042859

6. Anoje C, Aiyenigba B, Suzuki C, Badru T, Akpoigbe K, Odo M, et al. Reducing mother-to-child transmission of HIV: findings from an early infant diagnosis program in south-south region of Nigeria. BMC Public Health (2012) 12:184. doi: 10.1186/1471-2458-12-184

7. Nkenfou CN, Lobé EE, Ouwe-Missi-Oukem-Boyer O, Sosso MS, Dambaya B, Gwom LC, et al. Implementation of HIV early infant diagnosis and HIV type 1 RNA viral load determination on dried blood spots in Cameroon: challenges and propositions. AIDS Res Hum Retrovirus. (2012) 28:176-81. doi: 10.1089/aid.2010.0371

8. World Health Organization. Guidance on Global Scale-Up of the Prevention of Mother-to-Child Transmission of HIV. Towards Universal Access for Women, Infants and Young Children and Eliminating HIV and AIDS Among Children, With The Interagency Task Team (IATT) on Prevention of HIV Infection in Pregnant Women, Mothers and their Children. Geneva: World Health Organization (2007), p. 1-40. Available online at: http://www.who.int/hiv/ pub/toolkits/PMTCT9789241596015_eng.pdf
Msingwa, and Diana Kwetukia. Thanks to Dr. Susan F. Rumisha and Coleman Kishamawe for their assistance in statistical analysis. Thanks to Dr. Juma R. Mfanga (District Medical Officer), Dorothy Lema (Regional Reproductive and Child Health Coordinator), Angelina D. Sengwaji (District Reproductive and Child Health Coordinator), Paulo B. Muhusa (District Laboratory Technologist), Herieth Nyangasa (Council HIV/AIDS coordinator-CHAC), John Henry Kwingwa (Community Development officer), Dr. Baltazar Nyombi (Head of the KCMC clinical laboratory) and staff of the Muheza Designated District Hospital (Teule Hospital), health centers and dispensaries for their extended cooperation during data collection. Many thanks to all children and their families who agreed to participate in this study. Thanks to the Education, Audio-visual and Culture Executive Agency Project of the European Commission for the support through the Intra-ACP (Africa, Caribbean, and Pacific) Scholarship offered to VB.

\section{SUPPLEMENTARY MATERIAL}

The Supplementary Material for this article can be found online at: https://www.frontiersin.org/articles/10.3389/fpubh. 2018.00139/full\#supplementary-material

9. Buchanan AM, Dow DE, Massambu CG, Nyombi B, Shayo A, Musoke R, et al. Progress in the prevention of mother to child transmission of HIV in three regions of Tanzania: a retrospective analysis. PLoS ONE (2014) 9:e88679. doi: 10.1371/journal.pone.0088679

10. Stevens W, Erasmus L, Moloi M, Taleng T, Sarang S. Performance of a novel human immunodeficiency virus (HIV) type 1 total nucleic acid-based realtime PCR assay using whole blood and dried blood spots for diagnosis of HIV in infants. J Clin Microbiol. (2008) 46:3941-5. doi: 10.1128/JCM.00754-08

11. Sherman GG, Cooper PA, Coovadia AH, Puren AJ, Jones SA, Mokhachane M, et al. Polymerase chain reaction for diagnosis of human immunodeficiency virus infection in infancy in low resource settings. Pediatr Infect Dis J. (2005) 24:993-7. doi: 10.1097/01.inf.0000187036.73539.8d

12. Ministry of Health and Social Welfare. The United Republic of Tanzania, National Guidelines for Comprehensive Care Services for Prevention of Mother to Child Transmission of HIV and Keeping Mothers Alive. Dar es Salaam: Ministry of Health and Social Welfare (2013), p. 1-172. Available online at: http://ihi.eprints.org/3335/1/tz_guidelines_ccs_optionb_all.pdf

13. World Health Organization. Fact Sheet. HIV Treatment and Care. What's New in Infant Diagnosis. World Health Organization (2015), p. 1-2. Available online at: http://41.77.4.165:6510/apps.who.int/iris/bitstream/10665/204346/ 1/WHO_HIV_2015.43_eng.pdf

14. Chilongozi D, Wang L, Brown L, Taha T, Valentine M, Emel L, et al. Morbidity and mortality among a cohort of human immunodeficiency virus type 1-infected and uninfected pregnant women and their infants from Malawi, Zambia, and Tanzania. Pediatr Infect Dis J. (2008) 27:804-81. doi: 10.1097/INF.0b013e31817109a4

15. World Health Organizatiion. Global Health Observatory Data. Number of People (All Ages) Living With HIV Estimates by Country. World Health Organizatiion (2017). Available online at: http://apps.who.int/gho/data/view. main.22100?lang=en

16. Nuwagaba-Biribonwoha H, Wang C, Kilama B, Jowhar FK, Antelman G, Panya MF, et al. Implementation of antiretroviral therapy guidelines for under-five children in Tanzania: translating recommendations into practice. Int AIDS Soc. (2015) 18:20303. doi: 10.7448/IAS.18.1.20303

17. Ciaranello AL, Park J-E, Ramirez-Avila L, Freedberg KA, Walensky RP, Leroy V. Early infant HIV-1 diagnosis programs in resource-limited settings: 
opportunities for improved outcomes and more cost-effective interventions. BMC Med. (2011) 9:59. doi: 10.1186/1741-7015-9-59

18. Ministry of Health and Social Welfare. The United Republic of Tanzania, National AIDS Control Program, National Guidelines for the Management of HIV and AIDS. Dar es Salaam: Ministry of Health and Social Welfare (2015).

19. Ministry of Health, Community Development, Gender, Elderly and Children. The United Republic of Tanzania, National PMTCT Programme Data. Ministry of Health, Community Development, Gender, Elderly and Children. Dar es Salaam (2017).

20. Chiduo MG, Mmbando BP, Theilgaard ZP, Bygbjerg IC, Gerstoft J, Lemnge M, et al. Early infant diagnosis of HIV in three regions in Tanzania; successes and challenges. BMC Public Health (2013) 13:910. doi: 10.1186/1471-2458-13-910

21. Cook RE, Ciampa PJ, Sidat M, Blevins M, Burlison J, Davidson MA, et al. Predictors of successful early infant diagnosis of HIV in a rural district hospital in Zambezia, Mozambique. J Acquir Immune Def Syndrom. (2011) 56:e104-109. doi: 10.1097/QAI.0b013e318207a535

22. Sutcliffe CG, van Dijk JH, Hamangaba F, Mayani F, Moss WJ. Turnaround time for early infant HIV diagnosis in rural Zambia: a chart review. PLoS ONE (2014) 9:e87028. doi: 10.1371/journal.pone.0087028

23. Mugambi ML, Deo S, Kekitiinwa A, Kiyaga C, Singer ME. Do diagnosis delays impact receipt of test results? Evidence from the HIV early infant diagnosis program in Uganda. PLoS ONE (2013) 8:e78891. doi: 10.1371/journal.pone.0078891

24. Noubiap JJN, Bongoe A, Demanou SA. Mother-to-child transmission of HIV: findings from an Early Infant Diagnosis program in Bertoua, Eastern Cameroon. Pan Afr Med J. (2013) 15:65. doi: 10.11604/pamj.2013.15.65.2551

25. Tejiokem MC, Faye A, Penda IC, Guemkam G, Ateba Ndongo F, Chewa G, et al. Feasibility of early infant diagnosis of HIV in resource-limited settings: the ANRS 12140-PEDIACAM study in Cameroon. PLoS ONE (2011) 6:e21840. doi: 10.1371/journal.pone.0021840

26. Nuwagaba-Biribonwoha $H$, Werq-Semo B, Abdallah A, Cunningham A, Gamaliel JG, Mtunga S, et al. Introducing a multi-site program for early diagnosis of HIV infection among HIV-exposed infants in Tanzania. BMC Pediatr. (2010) 10:44. doi: 10.1186/1471-2431-10-44

27. Bwana VM, Frimpong C, Simulundu E, Mfinanga SG, Mboera LE. Accessibility of services for early infant diagnosis of Human Immunodeficiency Virus in sub-Saharan Africa: a systematic review. Tanzania J Health Res. (2016) 18:1-18. doi: 10.4314/thrb.v18i3.9

28. Hassan AS, Sakwa EM, Nabwera HM, Taegtmeyer MM, Kimutai RM, Sanders EJ, et al. Dynamics and constraints of early infant diagnosis of HIV infection in rural Kenya. AIDS Behav. (2012) 16:5-12. doi: 10.1007/s10461-010-9877-7

29. Woldesenbet S, Goga AE, Jackson DJ, for the SA EID Study Group. The South African Programme to Prevent Mother-to-Child Transmission of HIV (PMTCT): Evaluation of Systems for Early Infant Diagnosis in Primary Health Care Facilities in South Africa: Report on Results of a Situational Assessment. Cape Town: South African Medical Research Council (2012).

30. Cherutich P, Inwani I, Nduati R, Mbori-Ngachad D. Optimizing paediatric HIV care in Kenya: challenges in early infant diagnosis. Bull World Health Organ. (2008) 86:155-60. doi: 10.2471/BLT.07.040402

31. Adeniyi VO, Thomson E, Ter Goon D, Ajayi IA. Disclosure, stigma of HIV positive child and access to early infant diagnosis in the rural communities of OR Tambo District, South Africa: a qualitative exploration of maternal perspective. BMC Pediatr. (2015) 15:98. doi: 10.1186/s12887-015-0414-8

32. Tanzania Commission for HIV and AIDS. United Republic of Tanzania, Country Progress Reporting for 2012, Part A: Tanzania Mainland. Dar-es- salaam (2012). Available online at: http://files.unaids.org/en/ dataanalysis/knowyourresponse/countryprogressreports/2012countries/ce TZ_Narrative_Report[1].pdf

33. National Bureau of Statistics. National Population Census: National Bureau of Statistics (2013). Available online at: http://www.nbs.go.tz/

34. Muheza District Council. Annual Primary Health Care Report. Muheza: Muheza District Council (2013).

35. Tanga Region. Annual Prevention of Mother to Child Transmission of HIV(PMTCT)Report. Tanga (2015).

36. Gorstein J, Sullivan K, Parvanta I, Begin F. Indicators and Methods for CrossSectional Surveys of Vitamin and Mineral Status of Populations. Atlanta, GA: Micronutrient Initiative (Ottawa) and the Centers for Disease Control (2007).
Available online at: www.who.int/vmnis/toolkit/mcn-micronutrient-surveys. pdf

37. Braun M, Kabue MM, McCollum ED, Ahmed S, Kim M, Aertker L, et al. Inadequate coordination of maternal and infant HIV services detrimentally affects early infant diagnosis outcomes in Lilongwe, Malawi. J Acquir Immun Defic Syndrom. (2011) 56:e122-8. doi: 10.1097/QAI.0b013e31820a7f2f

38. Joint United Nations Programme on HIV/AIDS. 2015 Progress Report On The Global Plan Towards the Elimination of New HIV Infections Among Children and Keeping Their Mothers Alive. Joint United Nations Programme on HIV/AIDS (2014), p. 7-21. Available online at: http://www.unaids.org/sites/ default/files/media_asset/JC2774_2015ProgressReport_GlobalPlan_en.pdf

39. Coulibaly M, Meda N, Yonaba C, Ouedraogo S, Congo M, Barry $\mathrm{M}$, et al. Missed opportunities for early access to care of HIVinfected infants in Burkina Faso. PLoS ONE (2014) 9:e111240. doi: 10.1371/journal.pone.0111240

40. Chetty T, Knight S, Giddy J, Crankshaw TL, Butler LM, Newell M-L. A retrospective study of Human Immunodeficiency Virus transmission, mortality and loss to follow-up among infants in the first 18 months of life in a prevention of mother-to-child transmission programme in an urban hospital in KwaZulu-Natal, South Africa. BMC Pediatr. (2012) 12:146. doi: 10.1186/1471-2431-12-146

41. Dube Q, Dow A, Chirambo C, Lebov J, Tenthani L, Moore M, et al. Implementing early infant diagnosis of HIV infection at the primary care level: experiences and challenges in Malawi. Bull World Health Organ. (2012) 90:699-704. doi: 10.2471/BLT.11.100776

42. Koye DN, Zeleke BM. Mother-to-child transmission of HIV and its predictors among HIV-exposed infants at a PMTCT clinic in northwest Ethiopia. BMC Public Health (2013) 13:398. doi: 10.1186/1471-2458-13-398

43. Mwendo EM, Mtuy TB, Renju J, Rutherford GW, Nondi J, Sichalwe AW, et al. Effectiveness of prevention of mother to child HIV transmission programmes in Kilimanjaro region, northern Tanzania. Trop Med Int Health (2014) 19:267-274. doi: 10.1111/tmi.12255

44. Ong'ech JO, Hoffman HJ, Kose J, Audo M, Matu L, Savosnick P, et al. Provision of services and care for HIV-exposed infants: a comparison of maternal and child health clinic and HIV comprehensive care clinic models. J Acquir Immun Def Syndrom. (2012) 61:83-9. doi: 10.1097/QAI.0b013e31825bd842

45. Wiegert K, Dinh T-H, Mushavi A, Mugurungi O, Kilmarx PH. Integration of prevention of mother-to-child transmission of HIV (PMTCT) postpartum services with other HIV care and treatment services within the maternal and child health setting in Zimbabwe, 2012. PLoS ONE (2014) 9:e98236. doi: 10.1371/journal.pone.0098236

46. Goodson JL, Finkbeiner T, Davis NL, Lyimo D, Rwebembera A, et al. Evaluation of using routine infant immunization visits to identify and followup HIV-exposed infants and their mothers in Tanzania. J Acquir Immun Def Syndrom. (2013) 63:e9-15. doi: 10.1097/QAI.0b013e31828a3e3f

47. Rollins N, Mzolo S, Moodley T, Esterhuizen T, van Rooyen H. Universal HIV testing of infants at immunization clinics: an acceptable and feasible approach for early infant diagnosis in high HIV prevalence settings. Aids (2009) 23:1851-7. doi: 10.1097/QAD.0b013e32832d84fd

48. McCollum ED, Johnson DC, Chasela CS, Siwande LD, Kazembe PN, Olson D, et al. Superior uptake and outcomes of early infant diagnosis of HIV services at an immunization clinic versus an "under-five" general pediatric clinic in Malawi. J Acquir Immun Def Syndrom. (2012) 60:e107-10. doi: 10.1097/QAI.0b013e31825aa721

49. Kankasa C, Carter RJ, Briggs N, Bulterys M, Chama E, Cooper ER, et al. Routine offering of HIV testing to hospitalized pediatric patients at university teaching hospital, Lusaka, Zambia: acceptability and feasibility. J Acquir Immun Def Syndrom. (2009) 51:202-8. doi: 10.1097/QAI.0b013e31819c173f

50. Ndondoki C, Brou H, Timite-Konan M, Oga M, Amani-Bosse C, Menan $\mathrm{H}$, et al. Universal HIV screening at postnatal points of care: which public health approach for early infant diagnosis in Côte d'Ivoire? PLoS ONE (2013) 8:e67996. doi: 10.1371/journal.pone.0067996

51. Creek T, Tanuri A, Smith M, Seipone K, Smit M, Legwaila K, et al. Early diagnosis of human immunodeficiency virus in infants using polymerase chain reaction on dried blood spots in Botswana's national program for prevention of mother-to-child transmission. Pediatr Infect Dis J. (2008) 27:226. doi: 10.1097/INF.0b013e3181469050 
52. Weigel R, Kamthunzi P, Mwansambo C, Phiri S, Kazembe PN. Effect of provider-initiated testing and counselling and integration of ART services on access to HIV diagnosis and treatment for children in Lilongwe, Malawi: a pre-post comparison. BMC Pediatr. (2009) 9:80. doi: 10.1186/1471-2431-9-80

53. Wanyenze RK, Nawavvu C, Ouma J, Namale A, Colebunders R, Kimya $\mathrm{MR}$, et al. Provider initiated HIV testing for paediatric inpatients and their caretakers is feasible and acceptable. Trop Med Int Health (2010) 15:113-9. doi: 10.1111/j.1365-3156.2009.02417

54. Ioannidis J, Taha TE, Kumwenda N, Broadhead R, Mtimavalye L, Miotti $\mathrm{P}$, et al. Predictors and impact of losses to follow-up in an HIV-1 perinatal transmission cohort in Malawi. Int J Epidemiol. (1999) 28:769-75. doi: $10.1093 /$ ije/28.4.769

55. Woldesenbet SA, Jackson D, Goga AE, Crowley S, Doherty T, Mogashoa M, et al. Missed opportunities for early infant HIV diagnosis: results of a national study in South Africa. J Acquir Immun Def Syndrom. (2015) 68:e26-32. doi: 10.1097/QAI.0000000000000460

56. Ahoua L, Ayikoru H, Gnauck K, Odaru G, Odar E, Ondoa-Onama C, et al. Evaluation of a 5-year programme to prevent mother-to-child transmission of HIV infection in Northern Uganda. J Trop Pediatr. (2010) 56:43-52. doi: 10.1093/tropej/fmp054
57. Jones S, Sherman G, Varga C. Exploring socio-economic conditions and poor follow-up rates of HIV-exposed infants in Johannesburg, South Africa. AIDS Care (2005) 17:466-70. doi: 10.1080/09540120412331319723

58. Bobrow EA, Yemaneberhan AG, Phiri M, Katirayi L, Ahimbisibwe A, Chimbwandira F, et al. Barriers, facilitators and recommendations for the early infant diagnosis and treatment (EIDT) cascade: a qualitative study in Malawi. South Afr J Child Health (2016) 10:116-20. doi: 10.7196/SAJCH.2016.v10i2.982

Conflict of Interest Statement: The authors declare that the research was conducted in the absence of any commercial or financial relationships that could be construed as a potential conflict of interest.

Copyright (c) 2018 Bwana, Mfinanga, Simulundu, Mboera and Michelo. This is an open-access article distributed under the terms of the Creative Commons Attribution License (CC BY). The use, distribution or reproduction in other forums is permitted, provided the original author(s) and the copyright owner are credited and that the original publication in this journal is cited, in accordance with accepted academic practice. No use, distribution or reproduction is permitted which does not comply with these terms. 